PREHOSPITAL CARE

\title{
A review of emergency equipment carried and procedures performed by UK front line paramedics on paediatric patients
}

\author{
K Roberts, F Jewkes, H Whalley, D Hopkins, K Porter
}

Emerg Med J 2005;22:572-576. doi: 10.1136/emj.2004.022533

See end of article for authors' affiliations

.....................

Correspondence to: Dr K Roberts, 77 Three Acres Lane, Dickens Heath, Solihull, Birmingham B90 1NZ, UK; jkroberts@doctors.org.uk

Accepted for publication 10 May 2005
Objectives: In 1997 a review of paramedic practice upon adult patients in the UK found many inconsistencies and deficiencies in basic care. A follow up review in 2002 identified widespread improvement in provision of equipment and skills to provide basic and advanced life support.

Paediatric care was not assessed in either review. The authors conducted this study to identify current standards of care in paediatric paramedic practice and areas of potential improvement.

Method: A questionnaire designed to determine what equipment and skills were available to paramedics for the management of common or serious paediatric emergencies was sent to chief executives of the 32 NHS Ambulance Trusts in England and Wales.

Results: The trend of expanding and standardising practice among adult patients has not extended to paediatric practice despite national guidelines from the Joint Royal Colleges Ambulance Liaison Committee (JRCALC). Furthermore there are some serious failings in the provision of care and skills. Many Trusts have not adopted JRCALC guidelines for the management of life threatening paediatric emergencies such as asthma, meningitis, and fluid replacement in hypovolaemia.

Conclusions: Ambulance Trusts not meeting standards set out in the JRCALC guidelines must address their areas of deficiency. Failure to do so endangers children's lives and leaves Trusts open to criticism.
$\mathrm{U}$ nited Kingdom NHS ambulance services aim to maintain life and alleviate patient morbidity until definitive care can be delivered in a hospital facility. Simple techniques and equipment are usually all that are required in order to achieve this, using the well rehearsed mantra of airway, breathing, and circulation. This is particularly true for children as the vast majority of paediatric deaths are due to hypoxia or hypovolaemic shock.

A study in $1997^{1}$ reviewing variations in equipment carried on UK front line ambulances concluded that basic levels of ambulance equipment were adequate but that some essential equipment was missing from the majority of Ambulance Trusts. Such equipment included nasopharyngeal airways (NPAs), Hudson type oxygen masks, traction splints, long boards, and vacuum splints. It was also suggested that UK paramedics should be able to perform needle cricothyroidotomy and needle thoracocentesis. The study was repeated in $2002,{ }^{2}$ four months following the introduction of the Joint Royal Colleges Ambulance Liaison Committee (JRCALC) guidelines, and found that the equipment available to UK paramedics and procedures that they may perform had expanded. Variation in practice between Trusts had decreased while the number of interventions had increased. Furthermore some Trusts demonstrated that they were keeping up to date with current medical thinking-for example, hypotensive resuscitation.

The current survey aimed to assess national practice upon paediatric patients and identify if the standardisation of equipment and skills by the ambulance services seen among adult patients is occurring in paediatric practice. The survey was commissioned on behalf of the research and development committee of the Faculty of Pre-Hospital Care at the Royal College of Surgeons in Edinburgh and follows the introduction of a new degree course for ambulance paramedics and the advent of paramedic protocols by JRCALC.

\section{METHOD}

In July 2003, a year and a half after publication of the second edition of JRCALC guidelines, each chief executive of the 32 NHS Ambulance Trusts in England and Wales was sent a postal questionnaire. No reminders were sent.

The questionnaire was designed to determine what equipment and skills were available to paramedics for the management of common or serious paediatric emergencies including basic and advanced life support. Questions were structured to follow the standard paradigm of the "primary survey"-airway, breathing, and circulation with haemorrhage control. Specific questions relating to extrication equipment, spinal immobilisation, analgesia, and specific paediatric emergencies were included.

\section{RESULTS}

Twenty two (69\%) Trusts replied to the survey. The results, including comparison with adult practice in 2002 where appropriate (indicated as "adult \% of Trusts"), are presented in tables 1 to 5 .

In tables and text "Trusts (\%)" refers to the percentage of Trusts who responded to the questionnaire and not the total number of UK NHS Ambulance Trusts.

\section{Airway}

Table 1 shows the equipment and procedures available to the paramedic for airway control. One Trust cannot provide high flow Oxygen (12-15 l/min) because they do not carry paediatric non re-breather masks with a reservoir bag. All trusts carry oropharyngeal airways. Only $27 \%$ of Trusts permit the cutting of endotracheal tubes for use as nasopharyngeal airways. Intubation is permitted by all trusts,

Abbreviations: JRCALC, Joint Royal Colleges Ambulance Liaison Committee; LMA, laryngeal mask airway; NPA, nasopharyngeal airways; OPA, oropharyngeal airway. 
Table 1 Airway management

\begin{tabular}{lccc}
\hline $\begin{array}{l}\text { Equipment carried or } \\
\text { procedure performed }\end{array}$ & Trusts $(\mathbf{n})$ & Trusts (\%) & $\begin{array}{l}\text { Adult \% of } \\
\text { Trusts }\end{array}$ \\
\hline $\begin{array}{l}\text { Oropharyngeal airway } \\
\text { Nasopharyngeal airway* }\end{array}$ & 22 & 100 & 100 \\
$\begin{array}{l}\text { Intubation† } \\
\text { Neonatal straight }\end{array}$ & 22 & 27 & 55 \\
endotracheal blade & 14 & 100 & 100 \\
$\begin{array}{l}\text { Uncuffed endotracheal } \\
\text { tube }\end{array}$ & 20 & 64 & - \\
Laryngeal mask airway & 3 & 91 & - \\
Capnography & 3 & 14 & 26 \\
$\begin{array}{l}\text { Hudson type mask } \\
\text { Oxygen 12-15 I/min }\end{array}$ & 21 & 14 & - \\
Needle cricothyroidotomy & 13 & 95 & 94 \\
\hline
\end{tabular}

*In young children this necessitates cutting an endotracheal tube for use as an NPA.

†Eight Trusts do not permit intubation of younger children. The age limits are: under 1 year old (six Trusts), under 5 year old (one Trust), under 6 year old (one Trust)

however practice varies widely. $36 \%$ of Trusts do not carry straight blades (which are preferred for intubating very young children) and two do not carry uncuffed endotracheal tubes (preferred before puberty as the pressure of the cuff can damage the airway in these children). Six Trusts do not permit intubation of under one year olds, one Trust under five year olds, and one Trust under six year olds. Three Trusts (14\%) permit the use of the laryngeal mask airway in children and three have capnography widely available. Needle cricothyroidotomy is permitted by $60 \%$ of Trusts.

\section{Breathing}

Table 2 shows that $73 \%$ of Trusts allow their paramedics to perform needle thoracocentesis in children, comparable to adult practice $(68 \%)$. Three Trusts do not permit or have the equipment for pulse oximetry. Twenty one (95\%) Trusts carry peak flow meters. None carry spacer devices for the inhalation of commonly used asthma medications.

\section{Circulation}

Table 3 shows the equipment available for the treatment of shock. All trusts carry intravenous cannulae and all permit the use of large bore, size 14 gauge cannulae. Eighty two per cent carry intraosseous needles and 23\% permit placement of cannulae in the external jugular vein. Twenty one (95\%) Trusts employ JRCALC guidelines for the resuscitation of patients suffering haemorrhagic shock with $20 \mathrm{ml} / \mathrm{kg}$ being given as a first bolus. Eleven Trusts follow guidelines allowing a second bolus. Practice varies in the remaining Trusts from no further fluid to unlimited boluses. The use of crystalloids in paediatric and adult practice is comparable (Hartmans solution, $64 \%$ (this study) and 68\% (adult study 2002); normal saline, $77 \%$ (this study) and $71 \%$ (adult study 2002)) whereas the availability of colloids continues to decrease (Gelofusine, 18\% (this study) $v$ 39\% (adult study 2002) and 54\% (adult study 1997); Haemaccel, 9\% (this study) v 29\% (adult study 2002) and $72 \%$ (adult study 1997)). Eighteen Trusts have a protocol for fluid replacement of paediatric burn victims; however, the protocols vary widely ( see table 3 ). Sixty eight per cent of Trusts carry cling film or Waterjel (Water-Jel Technologies, Carlstadt, New Jersey, USA) for topical application to prevent dehydration of burnt tissue, further fluid loss, and pain relief.

\section{Disability, and spinal and limb immobilisation}

The use of AVPU as a tool to assess conscious level is universal. Fourteen Trusts also employ the use of the paediatric Glasgow Coma Scale.
Table 2 Paediatric breathing and ventilation management

\begin{tabular}{llll}
\hline $\begin{array}{l}\text { Equipment carried or } \\
\text { procedure performed }\end{array}$ & Trusts (n) & Trusts (\%) & Adult \% of Trusts \\
\hline Needle thoracocentesis & 16 & 73 & 68 \\
Pulse oximetry & 19 & 86 & 95 \\
Peak flow meter & 21 & 95 & - \\
Spacer device & 0 & 0 & - \\
\hline
\end{tabular}

Table 3 Paediatric circulation, haemorrhage control, and burn management

\begin{tabular}{lrrr}
\hline $\begin{array}{l}\text { Equipment carried or } \\
\text { procedure performed }\end{array}$ & Trusts (n) & Trusts (\%) & Adult \% of Trusts \\
\hline $\begin{array}{l}\text { Circulation management } \\
\text { Intravenous cannula }\end{array}$ & 22 & 100 & 100 \\
$\quad$ Large bore (14G) & 22 & 100 & 100 \\
$\quad \begin{array}{l}\text { External jugular vein } \\
\text { Intraosseous needles }\end{array}$ & 18 & 23 & - \\
Intravenous fluids & & 82 & - \\
$\quad$ JRCALC protocol* & 21 & 95 & - \\
$\quad$ Hartman's solution & 14 & 64 & 68 \\
$\quad$ Normal saline & 17 & 77 & 71 \\
$\quad$ Gelofusine & 4 & 18 & 39 \\
$\quad$ Haemaccel & 2 & 9 & 29 \\
Burn management & & & \\
$\quad$ Cling film or Waterjel & 15 & 68 & - \\
$\quad$ Fluid replacement† & 18 & 81 & - \\
Protocol & & & \\
\hline
\end{tabular}

*JRCALC protocol $=20 \mathrm{ml} / \mathrm{kg}$ bolus.

†15 JRCALC version 2 protocol [ $20 \mathrm{ml} / \mathrm{kg}$ bolus] (15 Trusts): nil if

$<5$ years old and $500 \mathrm{ml}$ if $>5$ years old (two Trusts); if systolic BP

$<90 \mathrm{mmHg}$ or transfer time $>1$ hour (one Trust).

Table 4 Disability, and spinal and limb immobilisation

\begin{tabular}{llcl}
\hline $\begin{array}{l}\text { Equipment carried or } \\
\text { procedure performed }\end{array}$ & Trusts (n) & Trusts (\%) & Adult \% of Trusts \\
\hline AVPU & 22 & 100 & - \\
Paediatric GCS & 14 & 64 & - \\
Adult GCS & 18 & 82 & - \\
Spinal board & 22 & 100 & 97 \\
Paediatric scoop & 2 & 9 & - \\
Traction splint & 11 & 50 & 74 \\
Box splint & 21 & 95 & 97 \\
Inflatable splint & 4 & 18 & 23 \\
Vacuum splint & 13 & 59 & 77 \\
Extrication device & 19 & 87 & 97 \\
(TED or KED) & & & \\
\hline GCS, Glasgow Coma Scale. &
\end{tabular}

All Trusts returning the questionnaire carry long spinal boards and $9 \%$ paediatric scoop stretchers. The use of traction splints (namely Donway, Sagar, or Thomas) is permitted by half of the Trusts (compared with $74 \%$ of adult practice). Every Trust uses either box or vacuum splints (or both) for immobilisation of long bone fractures. Extrication devices such as the Telford and Kendrick devices are widely available $(87 \%)$. These can also be used as a method of paediatric spinal immobilisation.

\section{Medication and analgesia}

All Trusts surveyed use entonox. The availability of nalbuphine continues $(77 \%)$ and no Trust, in accordance with version 2 of the JRCALC guidelines, permits the use of morphine. Simple analgesics (paracetamol or oral nonsteroidal anti-inflammatory drugs) are employed by half of 
Table 5 Medication and analgesia

\begin{tabular}{|c|c|c|c|}
\hline $\begin{array}{l}\text { Equipment carried or } \\
\text { procedure performed }\end{array}$ & Trusts (n) & Trusts (\%) & Adult $\%$ of Trusts \\
\hline \multicolumn{4}{|l|}{ Analgesia } \\
\hline Paracetamol/oral NSAID & 11 & 50 & - \\
\hline Entonox & 22 & 100 & 100 \\
\hline Nalbuphine & 17 & 77 & 71 \\
\hline Morphine & 0 & 0 & 10 \\
\hline \multicolumn{4}{|l|}{ Asthma } \\
\hline Salbutamol inhaler & 0 & 0 & - \\
\hline Salbutamol nebuliser & 22 & 100 & - \\
\hline Ipratroprium inh/neb & 0 & 0 & - \\
\hline Hydrocortisone & 4 & 18 & - \\
\hline \multicolumn{4}{|l|}{ Anaphylaxis } \\
\hline Adrenaline & 21 & 95 & - \\
\hline Chlorpheniramine & 2 & 9 & - \\
\hline Hydrocortisone & 7 & 32 & - \\
\hline \multicolumn{4}{|l|}{ Epilepsy } \\
\hline Diazepam - IV & 17 & 77 & - \\
\hline Diazepam - rectal & 22 & 100 & - \\
\hline \multicolumn{4}{|l|}{ Meningitis } \\
\hline Benzylpenicillin & 17 & 77 & - \\
\hline With no rash present & 4 & 18 & - \\
\hline \multicolumn{4}{|l|}{ Croup } \\
\hline Nebulised adrenaline & 4 & 18 & - \\
\hline Nebulised budesonide & 0 & 0 & - \\
\hline Dexamethasone & 0 & 0 & - \\
\hline \multicolumn{4}{|l|}{ Hypoglycaemia } \\
\hline Routine BM check & 21 & 95 & - \\
\hline Glucose - oral & 12 & 55 & - \\
\hline $10 \%$ Glucose - IV & 17 & 77 & - \\
\hline Glucagon & 22 & 100 & - \\
\hline
\end{tabular}

NSAID, non-steroidal anti-inflammatory.

Note: The previous studies reviewing adult practice did not assess treatment of medical conditions.

the Trusts. Every Trust permits the use of nebulised salbutamol in the treatment of asthma although none carries salbutamol inhalers. The protocols for the volumes of nebulised salbutamol vary widely (number of Trusts that adopt each protocol is given in parentheses): no maximum unless side effects become a problem (JRCALC guidline) (nine); $5 \mathrm{mg} \max$ (two); <1 year old $2.5 \mathrm{mg}$, >1 year old no $\max$ (three); nil to $<1$ year olds and various other doses for $>1$ year olds (five); $2.5 \mathrm{mg}$ as required (one); $5 \mathrm{mg}<5$ year old, $10 \mathrm{mg}>5$ year old (one); and no protocol (three). Hydrocortisone can be given by four Trusts (18\%) for the treatment of asthma.

One Trust does not allow the use of adrenaline (epinephrine) in anaphylaxis whereas two (9\%) permit chlorphenamine (not recommended in version 2 but is now in version 3 of the guidelines) and seven (32\%) hydrocortisone.

All Trusts carry diazepam for rectal administration and 17 $(77 \%)$ permit the use of intravenous diazepam. Version 3 of the guidelines permits intravenous or rectal routes for the administration of diazepam.

Benzylpenicillin is carried by 17 (77\%) Trusts for the use in cases of suspected meningitis; four of these permit its use when there is no non-blanching purpuric rash.

Four Trusts permit the use of nebulised adrenaline in croup. None carries nebulised budesonide or oral dexamethasone.

One Trust does not routinely check finger tip blood sugar levels but all carry glucagon. Intravenous glucose is carried by 17 Trusts $(77 \%)$ and oral by $12(55 \%)$.

\section{DISCUSSION}

This review demonstrates that paediatric care by UK paramedics is limited by resource and skill availability and on occasion accepted standards are seriously lacking, as assessed by comparison with national guidelines. It is not clear why the observed improvement in adult practice has not extended to paediatric practice.
Hypoxia is the commonest mechanism of death in children and the equipment required to maintain an airway is minimal, inexpensive, and easy to use. Standards of paediatric airway care are varied and on occasion inadequate. Every Trust returning a questionnaire approved the use of OPAs and allowed intubation in children but few encouraged the use of nasopharyngeal airways. In cases where an OPA is contraindicated, the NPA is a simple, safe, and potentially lifesaving alternative. Although smallest commercially available NPAs have a $5 \mathrm{~mm}$ internal diameter, many Trusts (73\%) are not using either these preformed tubes or making their own by cutting an appropriately measured endotracheal tube to size. This observation is not confined to paediatric practice. ${ }^{24}$ In 2002 only 55\% of Trusts carried NPAs for adult use. A suspected base of skull fracture is a widely taught contraindication for NPA placement and it is possible that fear of this complication has limited the use of the NPA. However the evidence for this complication is based upon a single case report ${ }^{5}$ and has recently been contested on the grounds that the clinical indicators of a basal skull fracture can be difficult to interpret, especially outside of the well lit hospital environment and that teaching should focus on correct placement of the NPA (parallel to the nasal floor) and the advantages of this piece of equipment rather than the often quoted but once cited complication. ${ }^{6}$

The value of prehospital intubation of children is dubious, with a large, well conducted study demonstrating no benefit over efficient bag valve mask ventilation. ${ }^{7}$ Nevertheless, paramedics should be able to place an endotracheal tube in a child of any age, in case of a difficult airway or special circumstances making bag valve mask ventilation less desirable - such as very long transit times or when there is a high risk of aspiration such as drowning. This skill is infrequently performed and therefore it is essential that all appropriate equipment should be available to make life as easy as possible. Age discrimination is unacceptable. Endotracheal intubation carries risks, the most important being unrecognised oesophageal intubation. The risk is increased when no anaesthetic agents are used, as is the case with UK paramedics. Procedures need to be in place to recognise this complication. ${ }^{8}$ One such procedure is the use of end tidal $\mathrm{CO}_{2}$ monitoring, which is recommended in the JRCALC guidelines, and available in only three Trusts.

While the laryngeal mask airway (LMA) is widely used in hospital paediatric practice, its use in prehospital care has not been established and expert bodies do not yet recommend its use in children. It may prove to provide a valuable mechanism of airway maintenance, as it is undoubtedly easier to place than an endotracheal tube. This device does not protect the patient from aspiration of gastric contents as effectively as an endotracheal tube; however, the incidence of aspiration in non-fasted patients has been vastly overestimated. ${ }^{9}$ In addition it is relatively easy to learn how to use and retain this skill. ${ }^{10}$

Cricothyroidotomy is an emergency procedure to "buy" time and is simple to perform. In children it could be lifesaving in cases of upper airway obstruction due to foreign body or epiglottitis and is a technique that every paramedic should be trained to perform, although skill decay is a concern. Furthermore, assembling equipment for the task can be cumbersome and take several minutes. The authors recommend constructing equipment and storing it in a safe place for use should an appropriate emergency arise.

Needle decompression of a tension pneumothorax, a simple and quick life saving procedure, can be performed by $73 \%$ of paramedics. This practice must become universal: tension pneumothorax can rapidly kill and prehospital diagnosis and treatment undoubtedly saves lives. Pulse oximetry provides rapid, non-invasive monitoring to assess 
the ability of the patient to oxygenate their blood and guide the paramedic as to the effectiveness and need for further airway or breathing support. It is less widely used in children than adults $(86 \% \vee 95 \%)$. The fact that it is less available to children than adults suggests that Trusts have failed to purchase paediatric sensors-presumably on grounds of economy. This is unacceptable, because the main cause of death in children is hypoxia.

All Trusts appropriately employ wide bore cannulae. Eighty two per cent carry the intraosseous needle and $23 \%$ allow placement of cannulae in the external jugular vein. In shocked patients venous access can be difficult and intraosseous needles offer rapid access to the vascular compartment. All drugs and intravenous fluids can be given by this route. It is a concern, as with NPAs, cricothyroidotomy, and chest decompression, that a proportion of Trusts do not permit their use. Similarly, they are easy to learn how to use, effective, and there is little skill decay. There are complications with their use such as extravasation, osteomyelitis, or growth plate damage and therefore teaching methods of safe placement in order to avoid complications is essential.

The external jugular vein is a large peripheral vein that is easily accessible. Cannulating this vein is easy to teach and again the focus must be how to avoid complications such as damage to pleura and other deep structures.

Circulatory support is essential in trauma care. There is much debate and research over volume and type of fluid that should be used in the resuscitation of adult patients in and outside of hospital. ${ }^{11-15}$ However children have very different physiological and compensatory mechanisms to hypovolaemic shock and the authors are not aware of any significant work investigating fluid resuscitation of children in prehospital care. It is important not to overly extrapolate adult conclusions to children who have different cardiovascular physiology.

The increased use of crystalloids and decreased use of colloids reveal that ambulance Trusts are adapting to the current trend in medical practice. A recent systematic review of randomised controlled trials comparing colloid and crystalloid resuscitation in critically ill trauma patients found no difference in outcome. ${ }^{15}$ A further systematic review of randomised controlled trials identified an increased relative risk of death associated with colloid use $(2.6,95 \%$ CI 1.1 to 5.9) in a similar group of patients. ${ }^{16}$ All this, however, is adult work. If crystalloid is to be given the choice is important. The use of a lactate containing solution (such as Hartman's) in massive haemorrhagic shock decreases acidosis and improves outcome in adults. ${ }^{12}$ However children metabolise lactate poorly, particularly in "medical" shock where lactic acidosis may become severe (for example, meningococcal sepsis) and it may be that solutions without lactate, such as $0.9 \%$ sodium chloride, are best.

Although the arguments continue over what fluid type is best it is accepted that warm fluid must be given to avoid secondary coagulopathy. ${ }^{17}$ This is particularly important in children as their body surface to volume ratio predisposes them to hypothermia.

A further trend adopted by prehospital practitioners in adult practice is "hypotensive resuscitation". This describes limited fluid replacement to achieve vital organ perfusion while accepting a lower than normal blood pressure in an attempt to control further blood loss. A detailed discussion is beyond the remit of this paper and can be found elsewhere. ${ }^{17}$ However, during haemorrhage, children maintain their blood pressure very well before rapid cardiovascular collapse and death. The hypotensive state that can be identified and then maintained by limited fluid replacement in adults is not easy to identify in children. The JRCALC (version 3) guidelines therefore recommend up to two $20 \mathrm{ml} / \mathrm{kg}$ fluid boluses aiming to normalise pulse and capillary refill time.

Fluid resuscitation outside of hospital can increase onscene time and the total volumes of fluid infused are low because of short scene and transit times. ${ }^{18} 19$ The temptation to cannulate on-scene and infuse fluids should be deferred in non-entrapped patients until the ambulance is en route to definitive care. If the patient is trapped and greater circulatory support is required prehospital then medical advice should be sought from prehospital doctors or hospital based medical personnel.

A minimum standard of care is the ability to splint fractures and immobilise the spine. This is achieved universally. Some trusts carry specific paediatric scoop stretchers or spinal boards; however the majority use adult long spinal boards-presumably using blankets to stabilise smaller children on the board.

Lower limb traction splints, such as Donway, Sagar, or Thomas, can be used by $50 \%$ of Trusts on paediatric patients. This is $24 \%$ less than adult practice. Low limb fractures, especially of the femur, cause moderate blood loss if the fracture is closed and massive haemorrhage if open. Traction splintage decreases the volume of blood loss and also the incidence of fat embolism and pain. The use of the Thomas splint in the First World War contributed to a decreased mortality from $80 \%$ to $8 \%$ in open fractures of the femur. ${ }^{20}$ It is appreciated that their application takes several minutes, but in cases of long on-scene or transport times their use can be life saving and certainly decreases morbidity and pain.

Four Trusts still use inflatable splints despite concerns over microvascular compromise. ${ }^{21} 22$ Now that box or vacuum splints are available this equipment should be abandoned.

Extrication devices such as the Telford Extrication Device (TED) and Kendrich Extrication Device (KED) can be very useful in the extrication of trapped patients from motor vehicle accidents. In children they have a further use-their design enables spinal immobilisation and a means of transport.

A long term criticism of the ambulance service has been the inadequate provision of analgesia. ${ }^{12}$ In adult practice this seems to be addressed, ${ }^{2}$ however the paediatric patient has until recently been exempt. At the time of the study paramedics were not permitted to give paediatric patients morphine. However, in 2003, the Medicines and Health care products Regulatory Agency (MHRA) approved its use. Version 3 (March 2004) of the JRCALC guidelines include advice on the use of morphine in children and this must be encouraged because of the distress and physiological stress that pain causes children. When doses are calculated for the patient's age the response to intravenous morphine is predictable and safe.

With the paramedic role continuing to expand and the introduction of a paramedic degree course, there is scope to consider the use of other forms of anaesthesia. One suitable example would be the use of local anaesthetic to anaesthetise the femoral nerve in fractures of the femur. This aids pain relief, splintage, and transportation of the patient. Another would be the use of ketamine to aid extrication of patients with fractured limbs trapped in road traffic accidents. Furthermore younger children do not appear to experience unpleasant emergence phenomena, which can be a problem with its use in adults.

Every Trust carries salbutamol for nebulised administration, however the doses given to patients vary greatly and three Trusts do not allow administration to under one year olds. Once more discrimination on the basis of age is unacceptable. At the time of the study ipratroprium was not permitted, this has been changed in version 3 of the 
guidelines. Salbutamol is less effective in under one year olds whereas ipratropium can be particularly effective.

This study has identified many areas of deficiency; however, there are infrequent but encouraging examples of Trusts permitting more advanced practice than basic guidelines recommend. Regarding the treatment of asthma, four Trusts permit the use of hydrocortisone (not discussed in JRCALC guidelines). Another, and more contentious, example is the administration of intravenous benzylpenicillin in cases of suspected meningitis. Currently the majority of Trusts (76\%) adopt JRCALC guidelines and permit this when a patient's history is in keeping with meningitis and the patient has a non-blanching purpuric rash. Antibiotic therapy is more effective given earlier in the disease, before rash formation, and four Trusts permit the administration of benzylpenicillin when there is no rash but the history is suggestive of meningitis. Presumably this is by a locally arranged patient group directive, a legal arrangement permitting non-doctors to prescribe in certain circumstances. This attitude of widening the paramedics scope to improve patient care and to expand practice has to be encouraged, although there are concerns over the diagnostic abilities of paramedics and thus inappropriate administration of benzylpenicillin with potential serious adverse reaction. We would advise Trusts to support their paramedics with additional, specific training when establishing such patient group directives. Audit of this practice is essential along with dissemination of results with other Trusts.

The management of anaphylaxis varies widely. One Trust does not even allow the administration of intramuscular adrenaline whereas two permit chlorpheniramine and seven hydrocortisone. At the time of the study, chlorpheniramine was not endorsed by the JRCALC guidelines but has been in the current version. Although hydrocortisone and chlorphenamine have a delayed onset of action, their administration before hospitalisation is desirable if transfer is not delayed by the process.

\section{CONCLUSION}

The trend of expanding and standardising practice among adult patients has largely not extended to paediatric practice, despite national guidelines being in place for over 18 months. Basic standards of care are widely achieved. Such practice includes the use of high flow oxygen combined with suitable oxygen masks, simple airway adjuncts including the ability of modify endotracheal tubes for the use as NPAs, the ability to gain IV access and give fluid, spinal immobilisation, and fracture management. However it is not adequate that such basic care is widely practiced-universal practice must be achieved. Furthermore age discrimination is not acceptable. Ambulance Trusts not meeting standards set out in the JRCALC guidelines must address their areas of deficiency. Failure to do so endangers children's lives and leaves Trusts open to criticism. We recommend universal ability to provide or perform:

- high flow oxygen

- NPAs for any age of patient

- intubation equipment (including straight blades) for all ages

- cricothyroidotomy

- chest decompression

- pulse oximetry

- intraosseous access

- low limb traction splints

- morphine administration.
The management of common medical and traumatic emergencies is not universal and we recommend that Trusts streamline protocols and base them upon the JRCALC guidelines. Such cases include fluid replacement in trauma and burns and the management of asthma, anaphylaxis, and suspected meningitis. We also recommend wider use of the LMA and capnography.

Paramedics are under increasing pressure to perform a wider spectrum of skills and further existing standards of care. In this environment skill decay is a real problem, especially with infrequently performed but potentially life saving procedures. Paramedics must be fully supported to avoid this problem. Methods include Trusts developing regular in-house training sessions and the establishment of external training and review.

\section{Authors' affiliations}

K Roberts, Walsall Manor Hospital, West Midlands, Warwickshire and Northamptonshire Air Ambulance, UK

F Jewkes, Wiltshire Ambulance Service, UK

H Whalley, SHO Surgery, Heartlands Hospital, Birmingham, UK

D Hopkins, Warwickshire and Northamptonshire Air Ambulance, UK

K Porter, Selly Oak Hospital, Birmingham, UK

Competing interests: none declared

\section{REFERENCES}

1 Porter K, Allison K, Greaves I. Variations in equipment on UK front line ambulances. Pre-hospital Immediate Care 2000;4:126-31.

2 Roberts K, Allison K, Porter K. A review of emergency equipment carried and procedures performed by U.K. front line paramedics. Resuscitation 2003;58:153-8.

3 Cooke MW. How much to do at the accident scene? BMJ 1999;319:1150.

4 Allison K, Porter K. Nasopharyngeal airways: an under-utilised pre-hospital resource. Pre-hospital Immediate Care 2000;4:192-3.

5 Muzzi DA, Losasso TJ, Cucchiara RF. Complication from a nasopharyngeal airway in a patient with a basilar skull fracture. Anaesthesiology 1991;74:366-8.

6 Roberts K, Porter K. How do you size a nasopharyngeal airway? Resuscitation 2003;56:19-23.

7 Gausche M, Lewis RJ, Strattons SJ, et al. Effect of out of hospital pediatric endotracheal intubation on survival and neurologic outcome. A controlled clinical trial. JAMA 2000;283:783-90.

8 Katz SH, Falk JL. Misplaced endotracheal tubes by paramedics in an urban emergency medical services system. Ann Emerg Medicine 2001;37:62-4.

9 Brimacombe JR, Berry A. The incidence of aspiration associated with the laryngeal mask airway: a meta-analysis of published literature. J Clin Anaesthesia 1995;7:297-305.

$10 \mathrm{Gwinnut}$ C. Alternatives to endotracheal intubation in airway management Journal of the British Association of Immediate Care 1996;19:37-41.

11 Revell M, Porter K. Pre-hospital fluids - when and how much? Trauma 2000;2:179-86.

12 Ho AM, Karmakar MK, Contardi LH, et al. Excessive use of normal saline in managing traumatized patients in shock: a preventable contributor to acidosis. J Trauma 2001;51:173-7.

13 Kwan I, Bunn F, Roberts I. Timing and volume of fluid administration for patients with bleeding following trauma. Cochrane Database Syst Rev 2001;(1):CD002245.

14 Bunn F, Roberts I, Tasker R, et al. Hypertonic versus isotonic crystalloid for fluid resuscitation in critically ill patients. Cochrane Database Syst Rev 2000;(4):CD002045.

15 Alderson P, Schierhout G, Roberts I, et al. Colloids versus crystalloids for fluid resuscitation in critically ill patients. Cochrane Database Syst Rev $2001 ;(2): C D 001319$.

16 Choi PT, Yip G, Quinonez LG, et al. Crystalloids versus colloids in fluid resuscitation: a systematic review. Crit Care Med 1999;27:200-10.

17 Revell M, Greaves I, Porter K. Endpoints for fluid resuscitation in haemorrhagic shock. J Trauma 2003;54:Supp63-7.

18 Dalton AM. Prehospital intravenous fluid replacement in trauma: an outmoded concept? J R Soc Med 88:213P-16P.

19 Driscoll $P$, Kent A. The effect of scene time on survival. Trauma 1999; 1:23-30.

20 Taken from the profile of the life of Hugh Owen Thomas. Available at http:// www.surgical-tutor.org.uk (accessed 2 June 2005).

21 Christensen KS, Trautner S, Stockel M, et al. Inflatable splints: do they cause tissue ischaemia? Injury 1986;17:167-70.

22 Sloan JP, Dove AF. Inflatable splints-what are they doing? Arch Emerg Med 1984;1:151-5. 